\title{
PERFORMANCE TEST OF CANTILEVER TRANSFER STANDARD USED FOR AFM TIP CALIBRATION BASED ON ELECTROMAGNETIC COMPENSATION
}

\author{
J. Jiang ${ }^{1}$, H. Zhou ${ }^{2}$, Y. Zhang ${ }^{3}$, S. Wu $u^{4}$, Z. Zhang ${ }^{5}$, G. $\mathrm{Hu}^{6}$ \\ 1,3, 4, 5, 6 Laboratory of Force and Torque, Division of Mechanics and Acoustics, National Institute of Metrology, China, \\ ${ }^{1}$ jiangj1@nim.ac.cn, ${ }^{3}$ zhangye@ nim.ac.cn, ${ }^{4}$ wushi@nim.ac.cn, ${ }^{5}$ zhiminzhang@nim.ac.cn, ${ }^{6}$ hugang@nim.ac.cn \\ ${ }^{2}$ Laboratory of Photoelectric Detection and Image Processing, School of Precision Instrument and Opto-electronic \\ Engineering, Tianjin University, China, huiyan9519@tju.edu.cn
}

\begin{abstract}
:
This paper describes stiffness measurement of cantilever transfer standards used for Atomic Force Microscopy (AFM) tip calibration based on electromagnetic compensation. The transfer standard of cantilever is designed and manufactured based on the bulk fabrication of SOI wafers. The measure range of the transfer standard covers from $0.04 \mathrm{~N} / \mathrm{m}$ to $16 \mathrm{~N} / \mathrm{m}$. The series of transfer standard is designed for the calibration test of the cantilever used in AFM, along with the test apparatus specifically designed. The relative uncertainty of the stiffness is smaller than $2.4 \%(k=2)$.
\end{abstract}

Keywords: cantilever; stiffness; small force; electromagnetic compensation

\section{INTRODUCTION}

Atomic Force Microscopy (AFM) can be utilised to observe the matters under very small dimension with high sense of the force below $10^{-6} \mathrm{~N}$ [1-2]. The precise measurement depends on the spring constant of the cantilever and the displacement of the tip measured by positioning detector. In order to obtain the spring constant of the AFM tip, highaccuracy measurements of SI traceable small forces below millinewton have been realized by several NMIs [3-11]. Besides the small force standard, the standard cantilever also needs to be designed and manufactured so as to realize the stiffness measurement of AFM tip without being measured directly on the small force standard.

In this paper the design and the measurement of series of the cantilever transfer standard were presented with the small force standard based on electromagnetic compensation. The transfer standard was designed with a series of lengths for covering a wide range of stiffness and manufactured based on the bulk fabrication of SOI wafers. The uncertainty analysis of the stiffness measurement was also conducted. The stiffness of the transfer standard can be obtained with relative extended uncertainty of $2.4 \%(k=2)$. Along with the design of the test apparatus, the series of transfer standard can be used for the AFM tip calibration by pushing the cantilever to the transfer standard.

\section{DESCRIPTION OF THE TEST AND THE RESULTS}

The measurement of the series of the transfer standard was carried out on the measuring system based on the electromagnetic compensation balance. The cantilever tip can be precisely loaded to the weighing part, driven by a piezoelectric moving stage with a distance of $100 \mu \mathrm{m}$, along with the observation of the contact of tip and the loading part in different directions. Mounting to the clamp specially used in AFM, the tip can be loaded very similar to the working condition in the AFM. The structure of the measuring system is shown in Figure 1.

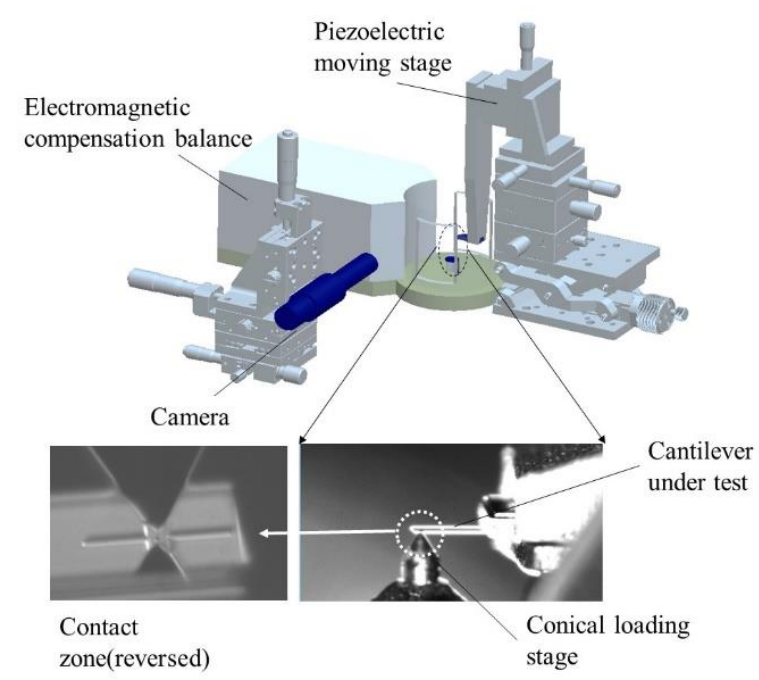

Figure 1: The structure of the measuring system

In Figure 2, instead of using the electromagnetic compensation balance, the transfer standard cantilever is applied to the measuring system. It is 
calibrated before assembly. This structure is still under construction. So with the help of this measuring system the transfer standard cantilever will be able to be utilised to calibrate the AFM tips without the complicated balance system.

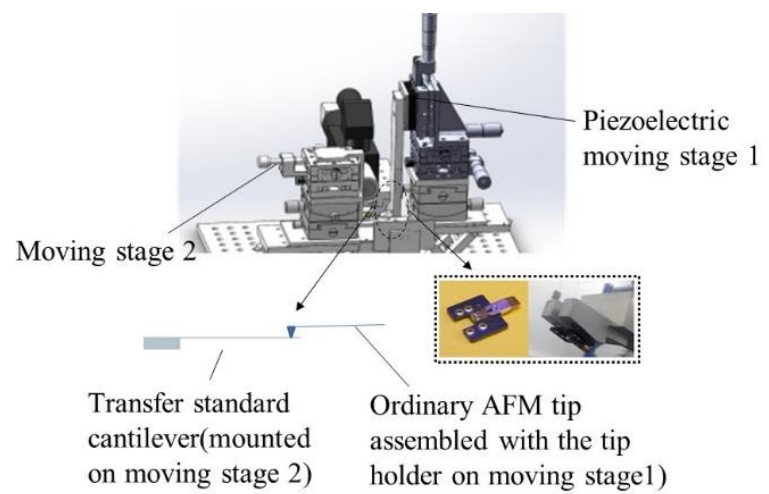

Figure 2: Design of the transfer standard test system

The transfer standard of cantilever is designed and manufactured based on the MEMS fabrication of SOI wafers. The effective lengths of the transfer standard are $L_{0}=8 \mathrm{~mm}, 7 \mathrm{~mm}, 6 \mathrm{~mm}, 5 \mathrm{~mm}, 4 \mathrm{~mm}$, $3 \mathrm{~mm}, 2 \mathrm{~mm}$, and $1 \mathrm{~mm}$. The width of the transfer standard is $w=500 \mu \mathrm{m}$. The nominal thickness is $t=0.01 \mathrm{~mm}$. This parameter is greatly influenced by the SOI wafer selected before the manufacture. The nominal stiffness of the transfer standard is $(0.041 \sim 21.125) \mathrm{N} / \mathrm{m}$. In the test all the cantilevers were loaded in a cycle with 11 steps of force, repeated at least 10 times. In each step the loading was maintained for $30 \mathrm{~s}$ to obtain the stable force. The temperature range is $21^{\circ} \mathrm{C}$ to $22^{\circ} \mathrm{C}$.
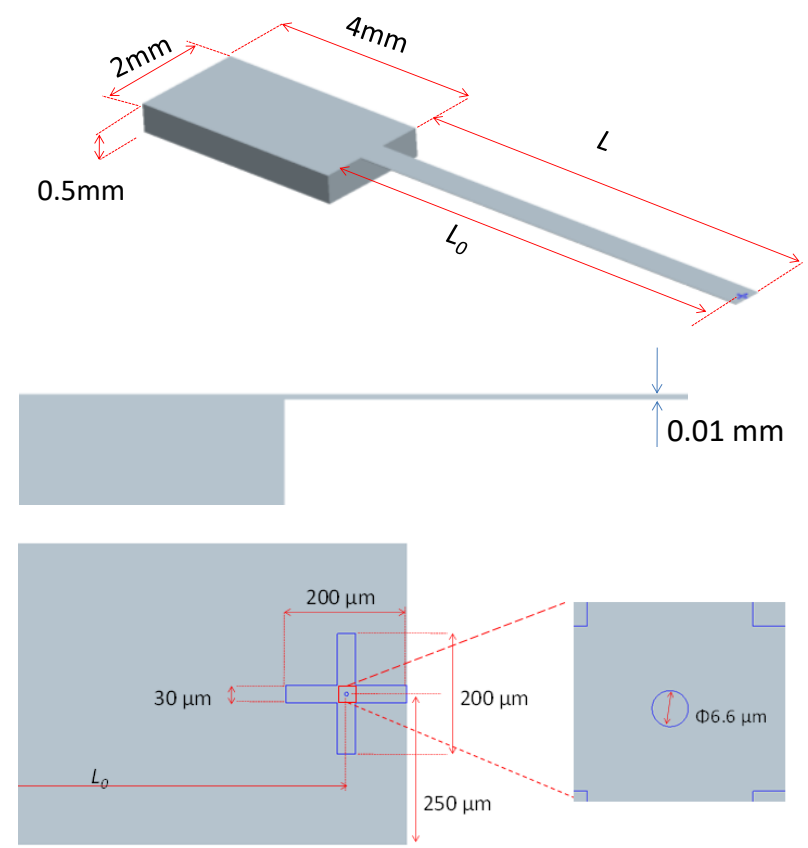

Figure 3: Design of the transfer standard and loading zone of transfer standard on the head of the tip

The loading part is assembled with conical diamond probe tip used in nano-indentation with a tip diameter of $1 \mu \mathrm{m}$ and the angle of $60^{\circ}$. This structure is for the test of the transfer standard. The typical test procedure is shown in Figure 4.

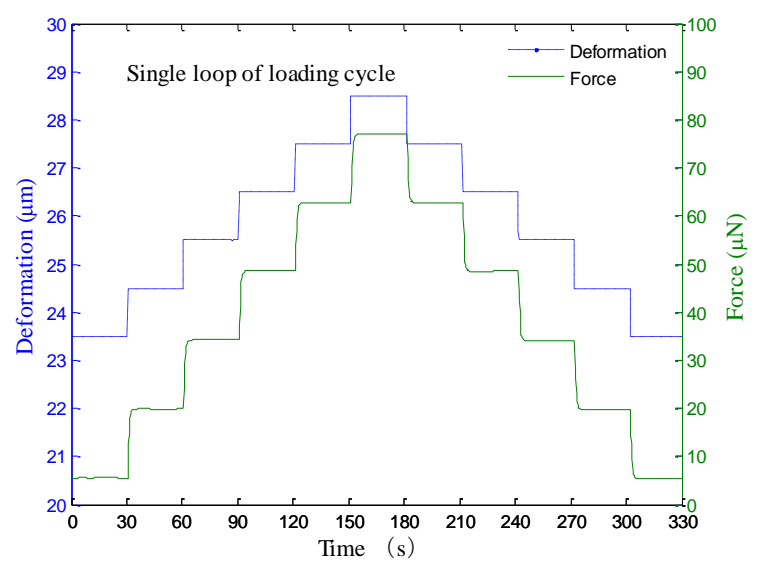

Figure 4: The test process of one loading cycle

Comparing to the theoretical design of the transfer standard cantilever, the actual stiffness is lower. According to the comparison shown in Figure 5, $10 \%-20 \%$ loss of the stiffness can be observed during the tests. Especially at the low range of the stiffness, the difference between the designed and the actual stiffness is more obvious, causing an exponential factor smaller than 1 in Figure 5 (bottom).
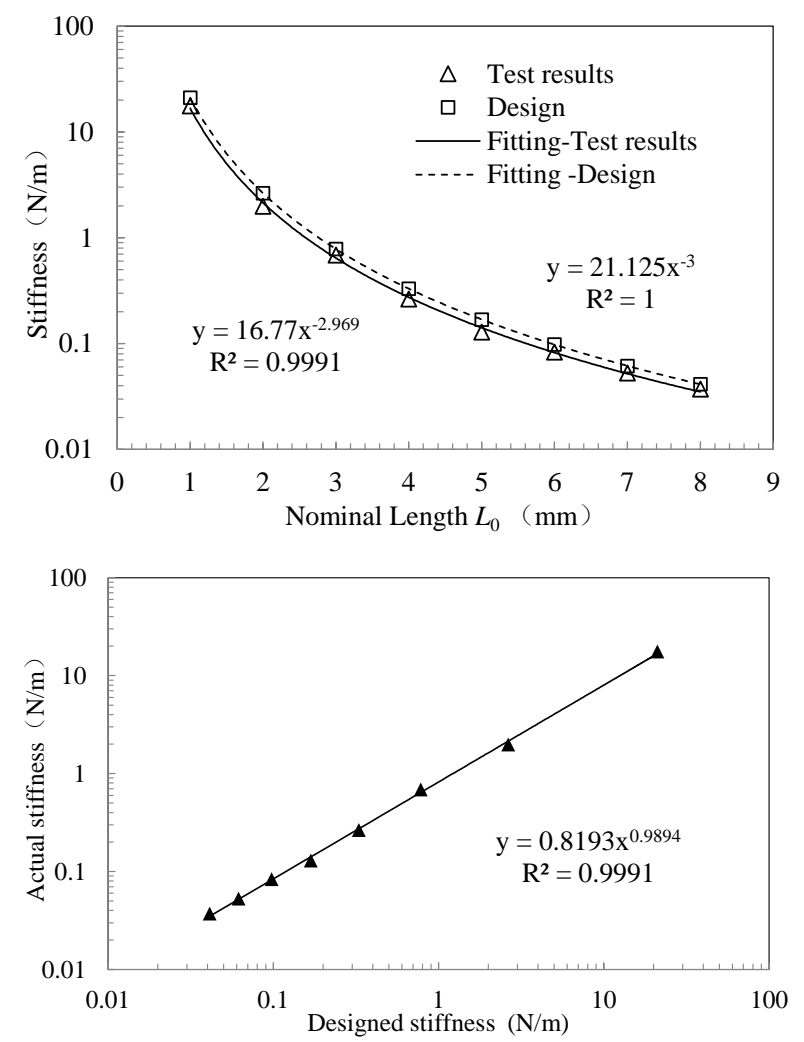

Figure 5: Difference between designed and the measured value of the stiffness (Top: Fitted analysis of the stiffness, Bottom: Deviation from the designed stiffness)

\section{UNCERTAINTY ANALYSIS}

The uncertainty analysis for the different type of the cantilevers was presented in references [12] and 
[13]. Here the uncertainty of the stiffness measurement for the transfer standard due to the repeatability $u_{1}$ is estimated by the standard deviation of the average value of measurement results in 10 cycles. The worst condition for the transfer standard is $0.97 \times 10^{-2}$ throughout the whole test. The other aspect regarding to uncertainty are originated from force and displacement measurements, non-linearity of calibration curves, orientation of the cantilevers, location accuracy of the measurement point and the stiffness of the measurement chain. The uncertainty due to the force measurement is estimated in [12]. The uncertainty originated from force $u_{2}$ is attributed to the uncertainty at the lower limit of the force.

The displacements of the nano-positioning stage were measured by a laser interferometer over $100 \mu \mathrm{m}$ with 20 steps of $5 \mu \mathrm{m}$. The standard uncertainty of the measurement results was $68.2 \mathrm{~nm}$. The relative uncertainty due to the displacement measurement overall the whole range within the measurement, $u_{3}$, is determined as $0.68 \times 10^{-4}$.

Derived from linear fit of force and corresponding displacement data, the residual of the linear fit is another source of the uncertainty, $u_{4}$, which is no more than $0.5 \times 10^{-2}$.

The stiffness of the cantilever is proportional to Young's modulus and the factors of cantilever dimensions. Therefore, the location of the contact point between the cantilever and the tip of the weighing pan should be considered. For the pin shown in Figure 3, the radius of the contact area is $3.3 \mu \mathrm{m}$. Assuming that $k=2.58$ (Gaussian distribution), the relative standard uncertainty due to location accuracy $u_{5}$ can be given by:

$$
u_{5}=\frac{1}{2.58} \frac{\delta L}{L}
$$

where $\delta L$ is the maximum position deviation from the ideal location. So $u_{5}$ is calculated to be $1.28 \times 10^{-3}$, where $\delta L=3.3 \mu \mathrm{m}, L=1000 \mu \mathrm{m}$.

The stiffness of the measurement chain $k_{\mathrm{b}}$ was determined to be $1099.1 \mathrm{~N} / \mathrm{m}$ [12]. Assuming a rectangular distribution, the relative standard uncertainty due to the stiffness of measurement chain $u_{6}$ is given by:

$$
u_{6}=\frac{1}{\sqrt{3}}\left|\frac{k_{\mathrm{m}}}{k_{\mathrm{b}}-k_{\mathrm{m}}}\right| \frac{\delta k_{\mathrm{b}}}{k_{\mathrm{b}}}
$$

where $\delta k_{\mathrm{b}}=120 \mathrm{~N} / \mathrm{m}$ is the maximum deviation of the stiffness of the measurement chain $k_{\mathrm{b}}$, and $k_{\mathrm{m}}$ is the stiffness of the cantilever in each measurement.

In the process of clamping the cantilever, we should also ensure that the balance probe and the central axis of the cantilever are in exact contact. Thus, the torque of the cantilever is 0 and the error caused by the torsion of the cantilever is eliminated.
However, in the actual operation process, it is difficult to ensure that the probe is just at the position of the central axis. It is known that the diameter of the probe used is $20 \mu \mathrm{m}$, the Young's modulus is $1140 \mathrm{GPa}$, and the Poisson's ratio is 0.07 . The cantilever Young's modulus $E$ is about $150 \mathrm{GPa}$, its Poisson's ratio $\mu$ is about 0.22 , and its shear modulus $G$ is given by:

$$
G=\frac{E}{2(1+\mu)}
$$

When the probe just aligns with the image of the cross centre of the cantilever, the probe is now on the cantilever boundary line, from which it can be seen that its displacement from the central axis of the cantilever is about $240 \mu \mathrm{m}$. According to the knowledge of material mechanics, its torsion angle $\phi$ is given by:

$\phi=\frac{12 T l}{G w t^{3}}$

The torque $T=F w / 2$, where $F$ is the force on the cantilever under the current position. Considering the maximum force $F$ of $200 \mu \mathrm{N}$ in the test of the cantilever with lowest stiffness, along with the length of the cross in the loading area of the cantilever, the width $w$ cannot be larger than $15 \mu \mathrm{m}$, deflection angle $\phi=0.032 \mathrm{rad}$, so the maximum uncertainty caused by torque angle is $0.13 \times 10^{-3}$. The influence can be omitted.

The combined standard uncertainty $u_{\mathrm{kc}}$ is determined by:

$u_{\mathrm{kc}}=\sqrt[2]{\sum_{i=1}^{6} u_{i}^{2}}$

The relative standard uncertainty is less than $1.2 \%$.

\section{CONCLUSION}

The cantilever transfer standard was fabricated based on bulk process and its stiffness has been measured based on small force standard based on electromagnetic compensation. The relative uncertainty of the stiffness is smaller than $2.4 \%$ $(k=2)$.

\section{ACKNOWLEDGEMENT}

This research is sponsored by National Key R\&D Program of China No.2018YFF0212401.

\section{REFERENCES}

[1] G. Binning, C. F. Quate, C. Gerber, "Atomic Force Microsope”, Phys. Rev. Lett., vol. 56, pp. 930-933, 1986. 
[2] N. A. Burnham, X. Chen, C. S. Hodges, et al, "Comparison of calibration methods for atomicforce microscopy cantilevers", Nanotechnology vol. 14, pp. 1-6, 2003.

[3] J. E. Sader, J. W. M. Chon, P. Mulvaney, "Calibration of rectangular atomic force microscope cantilevers", Review of Scientific Instruments vol. 70, pp. 3967-3969, 1999.

[4] B. Ohler, "Cantilever spring constant calibration using laser Doppler vibrometry", Review of Scientific Instruments, vol. 66, pp. 4583-4587, 1995.

[5] S. J. Chen, S. S. Pan, "A force measurement system based on an electrostatic sensing and actuating technique for calibrating force in a micronewton range with a resolution of nanonewton scale", Meas. Sci. Technol. vol. 22 pp. 045104, 2011.

[6] V. Nesterov, "Facility and methods for the measurement of micro and nano forces in the range below $10^{-5} \mathrm{~N}$ with a resolution of $10^{-12} \mathrm{~N}$ (development concept)", Meas. Sci. Technol. vol. 18, pp. 360-366, 2007.

[7] M. S. Kim, J. H. Choi, et al, "Accurate determination of spring constant of atomic force microscope cantilevers and comparison with other methods", Measurement, vol. 43 pp. 520-526, 2010.

[8] M. S. Kim, J. Pratt, U. Brand, C. W. Jones, "Report on the first international comparison of small force facilities: a pilot study at the micronewton level", Metrologia, vol. 49, pp. 70-81, 2012.

[9] C. A. Clifford, M. P. Seah, "The determination of atomic force microscope cantilever spring constants via dimensional methods for nanomechanical analysis", Nanotechnology, vol. 16, pp. 1666-1680, 2005.

[10] J. M. Neumeister, W. A. Ducker, "Lateral normal and longitudinal spring constants of atomic force microscopy cantilevers", Rev. Sci. Instrum., vol. 65, pp. 2527-2531, 1994.

[11] M. S. Kim, J. H. Choi， J. H. Kim, “SI-traceable determination of spring constants of various atomic force microscope cantilever with a small uncertainty of $1 \%$ ", Meas. Sci. Technol., vol. 18, pp.3351-3358, 2013.

[12] G. Hu, J. Jiang, Z. Zhang, Y. Zhang, U. Brand, M. S. Kim, "Investigation of a small force standard with the mass based method", Acta IMEKO, vol. 6, no. 2, pp.13-20, 2017.

[13] G. Hu, L. Song, F. Meng, W. Zhang, Z. Zhang, Y. Zhang, Y. Zheng, "Research and development of small force standards at NIM", Measurement of Mass, Force and Torque (APMF 2013) International Journal of Modern Physics: Conference Series, vol. 24, pp. 1360020-1-9, 2013.

[14] J. Wang, P. Fuchs, S. Russi, et al, "Uncertainty Evaluation for System of Weighing Equations for the Determination of Microgram Weights", IEEE Transactions on Instrumentation and Measurements, vol. 64, pp. 2272-2279, 2015. 